\title{
WiFi Data Acquisition System and online monitoring applied to thermoelectric microgeneration modules
}

\author{
R. I. S. Pereira ${ }^{1}$, P. C. M. Carvalho ${ }^{1}$ and S. C. S. Jucá ${ }^{2}$ \\ ${ }^{1}$ Electrical Engineering Department (DEE) \\ Federal University of Ceará - UFC \\ Fortaleza, 60455-760, Brazil \\ Phone/Fax number:+5585 33669585, e-mail: renata@dee.ufc.br, carvalho@dee.ufc.br \\ ${ }^{2}$ Telematics Area \\ Federal Institute of Ceará - Maracanaú Campus \\ Maracanaú, 61939-140, Brazil \\ Phone/Fax number: +5585 38786321, e-mail: sandrojuca@ifce.edu.br
}

\begin{abstract}
The present paper describes the development of an online monitoring and WiFi data acquisition system using free software applied to microgeneration based on thermoelectric modules. Monitoring and data acquisition systems are applicable in various stages of the microgeneration process, e.g., in the energy resource evaluation, generation failures prognosis, practical verification of project data and efficiency of generation. The online monitoring and data acquisition system was developed using a WiFi modem coupled to a microcontrolled board based on free software. This monitoring system was applied to thermoelectric modules and was also developed a wireless communication with the online server which sends microgeneration values to the data bank. The free software for online monitoring and WiFi data acquisition allows the analysis of stored data and charts through mobile devices as notebooks, tablets and smartphones. Moreover, the system can also be expanded to record values from analogical or digital sensors used in plants based on other renewable energy sources.
\end{abstract}

\section{Key words}

WiFi monitoring, Data acquisition systems, Thermoelectric microgeneration, Renewable energy sources.

\section{Introduction}

The thermoelectric microgeneration effect is an electric current flow when one junction of two dissimilar metals, joined at two places, was heated while the other junction was kept at a lower temperature [1]. Thermoelectric energy production is one of the main processes of converting thermal energy directly into electrical energy, and ensure a long reliable operation without maintenance due to the nonexistence of moving parts [2]-[7].

Thermoelectric modules (TEG), shown in Figure 1, can be formed by $\mathrm{P}$ and $\mathrm{N}$ type semiconductors which are connected in series electrically and parallel thermally among two ceramic layers. A TEG is made by heating one side and cooling the other side of the thermoelectric module and connecting a load to the module end points. As an environment-friendly energy source, thermoelectric power generation has attracted considerable interest due to its small size, zero carbon emission, absence of moving parts, low acoustic noise, high reliability and environmental energy production. Thermoelectric power generators are also a good choice to produce electric power by recycling waste thermal energy [8].

The TEG equipment used consists of four TEHP1-12640.8 thermoelectric modules connected in series, as illustrated in Figure 1, in order to obtain output voltage of $5 \mathrm{~V}$. The hot side of the module was attached to a metal base to support temperatures up to $250^{\circ} \mathrm{C}$. This base was then placed on the surface of the heat source by resistors. The modules are approximately $4 \mathrm{~cm} \mathrm{x} 4 \mathrm{~cm}$.

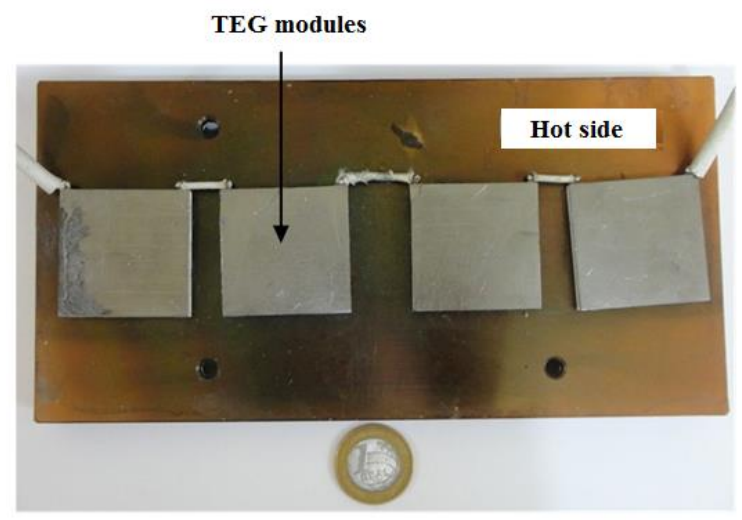

Fig. 1. TEG modules (TEHP1-1264-0.8).

A typical multi couple thermoelectric power module is illustrated schematically in Figure 2 which depicts p-type and n-type semiconductor thermoelements connected in series by highly metal interconnects strips to form a thermocouple [9]. 
Electrons on the hot side material are more energized than on the cold side. These electrons will flow from the hot to the cold side by connecting the both sides with a conductor wire. These electrons produce the electrical current. Connecting many thermocouples in series is possible to increase the output voltage and the output power.

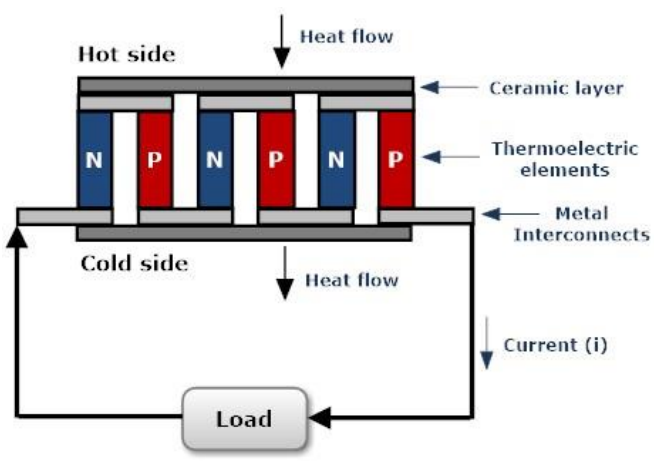

Fig. 2. Illustration of a thermoelectric microgeneration process.

The thermoelectric generators are also used by North American Space Agency (NASA) to supply electric energy to the Curiosity robot on Mars, through the heat liberated in radioisotope nuclear decay reactions [10], and as a source of renewable electric cogeneration through the heat supplied by incinerated municipal solid waste, automobile engines or industrial machinery [11].

Thermoelectric systems can be also designed either for using a big amount of energy (geothermal energy [12] for example) or for working with small heat sources, used in automotive or home applications for waste heat recovery. Thermoelectric modules have even been miniaturized to harvest body heat for powering a wristwatch [13]. Such small generators could be mass produced for home cogeneration of heat and electricity or used in automotive waste heat recovery. As shown in Figure 3, in a typical energy flow path of the internal combustion engine vehicle, only about $25 \%$ of the fuel combustion energy is utilized to propel the vehicles, whereas about $40 \%$ is wasted in the form of waste heat of exhaust gas [14]. It means that the fuel economy of internal combustion can be increased by up to $20 \%$ simply by capturing the waste heat of exhaust gas and converting about $10 \%$ of it to electricity with thermoelectric modules [15].

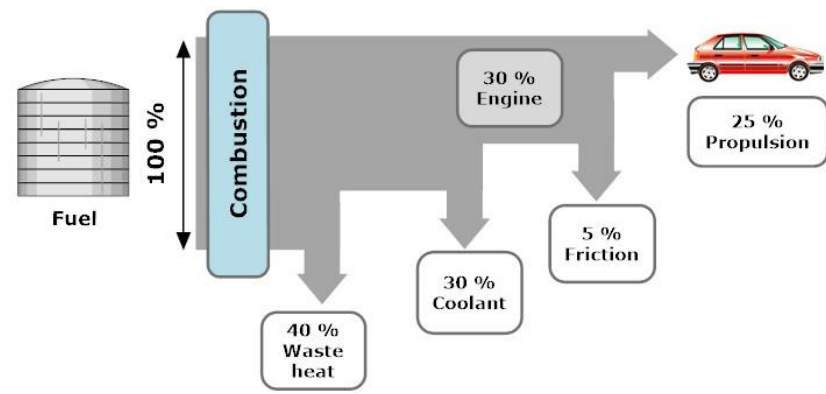

Fig. 3. Typical energy flow path in an internal combustion engine vehicle.

In this context, the present paper describes an online monitoring and WiFi data transmission embedded system for measurement of TEG modules efficiency.

\section{WiFi Acquisition System}

This section describes the developed data acquisition embedded system with WiFi connection, shown in Figure 4. The WiFi embedded system programming is based on open source and free software, which is an advantage of the proposed system. The board sends monitored data to an online server also programmed in open source software.

Data acquisition and online monitoring systems are found in Brazil mainly in large power plants, with complex monitoring and relatively high costs, making impracticable the implantation in domestic clients and in other clients that are inside the range of microgeneration power (up to $100 \mathrm{~kW}$ ). In this way, the present paper intends to develop efficient techniques for online monitoring with open source software, sensing and WiFi data transmission to contribute with the diffusion and installation of microgeneration systems based on renewable energy sources in Brazil so as thermoelectric microgeneration.

This modulated hardware consists of a microcontrolled board, called as SanUSB, connected to an WiFi RN-XV modem via an adapter board that was developed for this application in order to adjust the pin connections, as well as to convert the voltage from $5 \mathrm{~V}$ to $3.3 \mathrm{~V}$.

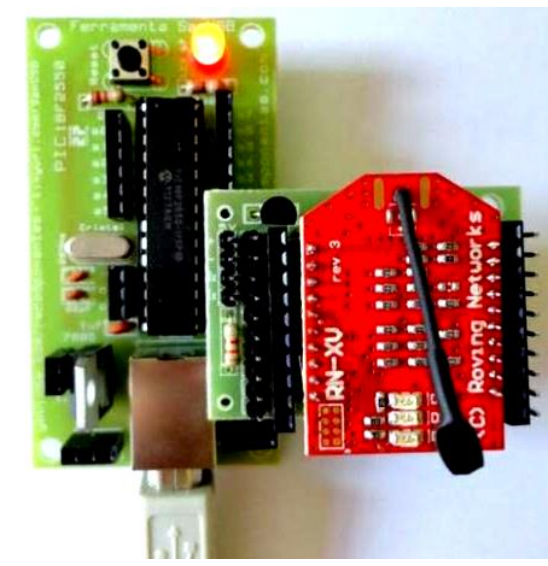

Fig. 4. Acquisition data board and WiFi modem

According to [16], a WiFi based platform was developed to collect also, data from human motion sensors and send over wireless connection to a mobile device in order to control interactive systems, i.e., musical applications.

Moreover, other example is a bioimpedance device for sarcopenia evaluation, i.e. age related muscle mass loss, based on WiFi protocol for the device remote control and allowing full integration to the Internet was described in [17]. A wireless system using RF signal to power a temperature sensor for on-body temperature monitoring is shown in [18].

There are also other studies on remote wireless sensing, such as those developed by [19] and [20]. Wireless sensor nodes inside buildings are used to read out sensor data and to control actuators by a reference value in [21]. The nodes need to operate for a long time with a single battery. When using a standard WiFi connection, the node battery 
would be depleted after a few hours due to idle currents in receive state. The use of sensor nodes with included wakeup receivers can prolong the lifetime of the sensor network to several years, because the sensor measure data and send information only when a command is received from the Internet.

The developed data acquisition embedded system, based in a microcontroller USB programmable tool and using open source software, is patented by National Institute of Industrial Property (INPI) with register number 088503 and executable in multiplatform like Linux, Mac OSX and Windows ${ }^{\circledR}$ with files available in SanUSB group [22]. Developed equipment for specific applications tend to be cheaper, have better cost benefit relation and make possible an easy understanding and operation. The mentioned open source software is based on contributions of Internet developers throughout the world. This software offers a better performance, encourages creativity, allows dedicated applications and stimulates finding and correcting code errors faster than private software [23]. It is important to emphasize that USB ports are used as power source by the tool microcontroller only in the code development phase.

In this context, the $\mathrm{WiFi}$ data transmission system for measurement of TEG modules efficiency consists in a hot side AC power controller (1), hot side thermocouple temperature sensor (2), data acquisition board (3) and WiFi modem (4) shown on Figure 5.

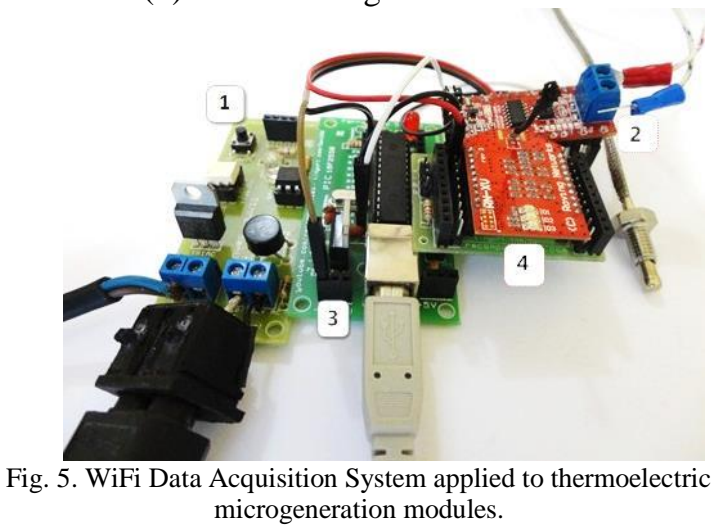

\section{TEG microgeneration monitoring}

WiFi nets use radio wave technology with Institute of Electrical and Electronics Engineers (IEEE) standards, such as IEEE 802.11a, 802.11b, and 802.11g. These standards provide reliable and safe wireless connectivity. $\mathrm{WiFi}$ nets can be used for connection between computational devices and also to connect these devices to Internet [24]. WiFi net operates in the not licensed radio waves $2.4 \mathrm{GHz}$, in the IEEE 802.11b and IEEE 802.11g technologies and in the $5 \mathrm{GHz}$ frequency of the $802.11 \mathrm{a}$ technology [25].

The WiFi modem is based in the $\mathrm{RN}-171$ module to promote connection to the wireless networks. The connection of this modem needs only four pins designated to power and WiFi communication. This device has an independent antenna to increase its reach and offers stronger signal and support for the most common communication protocols like Transmission Control Protocol (TCP), User Datagram Protocol (UDP), and File Transfer Protocol (FTP).

If more than one WiFi modem or computational device is connected on the Internet in a home network using a broadband router or a gateway, most of the time only this router will contain an Internet IP address and each device in the internal net utilizes an IP local address given by the router. The intranet IP local address is usually created dynamically via a service called Dynamic Host Configuration Protocol (DHCP) of the gateway, or defined in a static way by the user, according to the network. In the proposed case, the gateway is 192.168.1.1. Therefore, a fixed static IP address was defined for the WiFi embedded modem, i.e., 192.168.1.195. The proposed embedded system can be, with this firmware, server (e.g., switching a load through the instruction "192.168.1.195/YT" in address bar) or also client (e.g., a sensor value is posted to an online databank server).

In this case, information that is sent to the online data bank is configured with remote access over WiFi without charging, different from the applications that use General Packet Radio Service (GPRS) protocol. In this context, the development, programming and application of a WEB monitoring system and wireless data acquisition using open source software are described. The online data bank can be queried by any computational device connected to the Internet by using a personal password. The query can be made at any time, updated every minute. Figure 6 shows the online monitoring development in two stages:

1) Sensing, conditioning and data transmission;

2) Data bank uploading from an online server and presentation to the user.

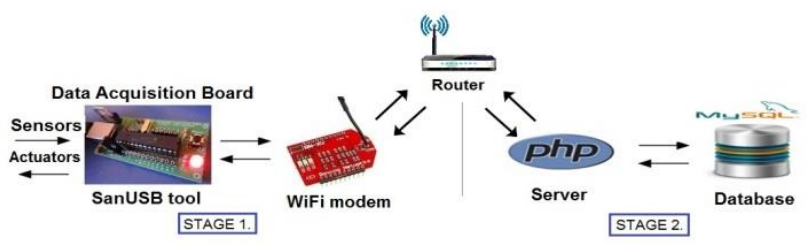

Fig. 6. Steps of the open source software monitoring system.

The first stage establishes communication between the sensors connected to the data acquisition board. The second stage, i.e., the user presentation layer, was developed in PHP with MySQL database. In this way, a free option for online monitoring applied to renewable energy sources is introduced. The first stage of sensing, conditioning, and wireless acquisition reads the data from the sensors every minute and saves the information in an internal Electrically-Erasable Programmable Read-Only Memory (EEPROM). Every ten minutes, the average value of the sensor measurement is calculated and sent to the stage two. It is important to mention that the actuators may be also connected to the board, allowing WiFi load control through the server. In the server, a communication interface to the acquisition system and another one working in parallel to the user communication were developed. The whole system is illustrated in Figure 7. 


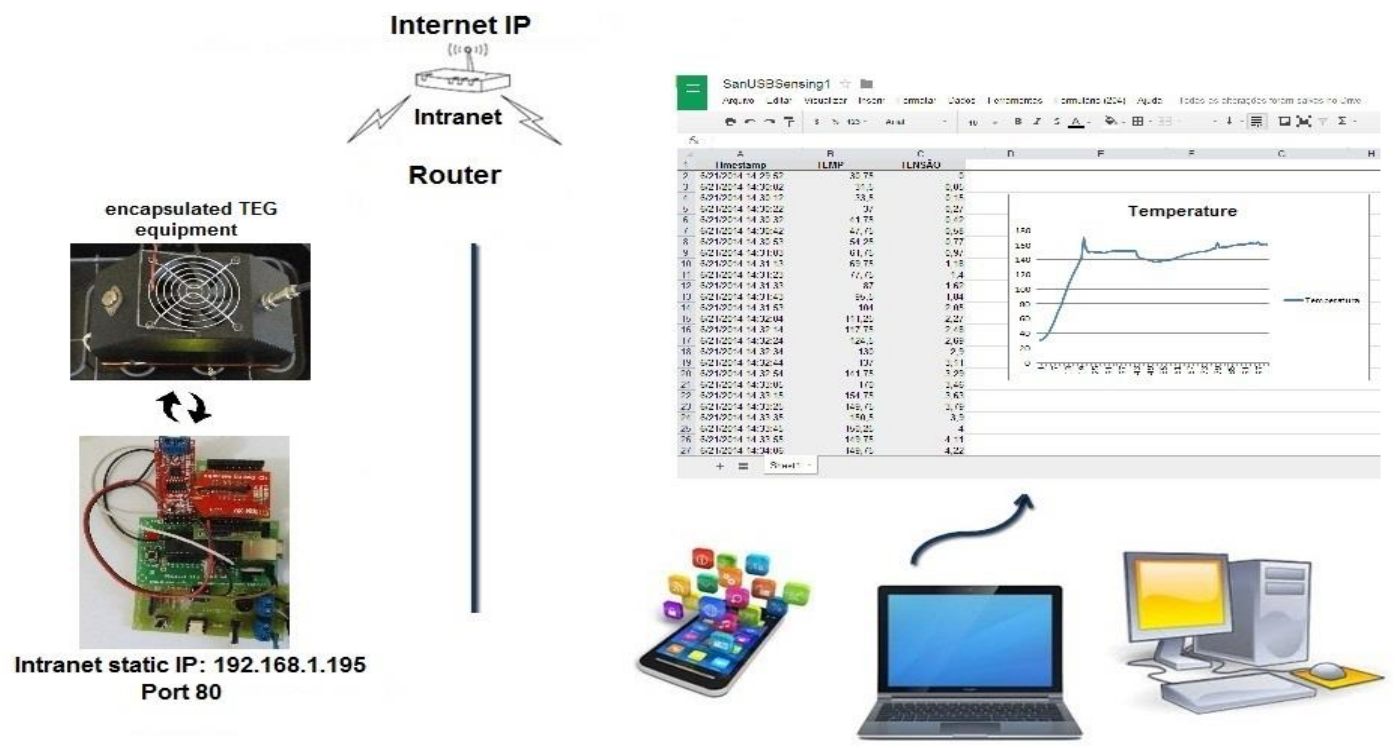

Fig. 7. Online monitoring process via WiFi modem.

The first interface is responsible for data receiving, to store them in the persistent database and to send a confirmation to the board. The user interface delivers a friendly front-end that allows data visualization in the form of list or chart. The embedded firmware was programmed using a digital filter to identify and exclude abnormal values that differ by more than $100 \%$ of the maximum and minimum values of the variable reference measured every minute.

The Web Monitor is an application developed on online server with the PHP programming language, in conjunction with a structured database in Database Management System (DBMS), in order to serve as visualization of monitoring data (signals sensors) from renewable energy sources and to send them via a wireless communication (WiFi). To access the monitoring system, it is necessary to enter the website link in PHP; after that, the system main authentication page appears, containing an authentication form (username and password). In this way, only pre-registered users can access the system. After entering the data access in the authentication step, the user is redirected to the system home page.

Through the WEB monitor, the user can query the stored data in the online database via smartphones or PC whereas the Ethernet standard is the solution currently used in private systems to promote network interconnectivity; the cost of monitoring systems for microgeneration in wireless network with open source software tends to be dwindling. The minimum sampling time for the online server viewing is approximately three seconds after sending data by WiFi modem, which is considered a limitation in real-time systems.

At the submenu Monitoramento (monitoring), there are two submenus: Gráfico (chart) and Logs, as shown in Figure 8. By clicking the submenu Gráfico a chart of the sensor values stored in the database is generated. To generate the charts, a library called Highcharts, written in Java Script, was used. The chart automatically restarts every 30 seconds. To display the sensors values involved in the chosen application in a specific day, it is necessary to inform or to select a valid date and then click the magnifying glass icon. Shortly after, the chart will be updated showing for each point a representative value averaged over the sensor values every 10 minutes. In order to view the corresponding logs to the current values displayed in the chart, it is necessary to click the logs button. The system also provides the option to print or export (PNG, JPEG, PDF, and SVG) the entire structure of the chart view.

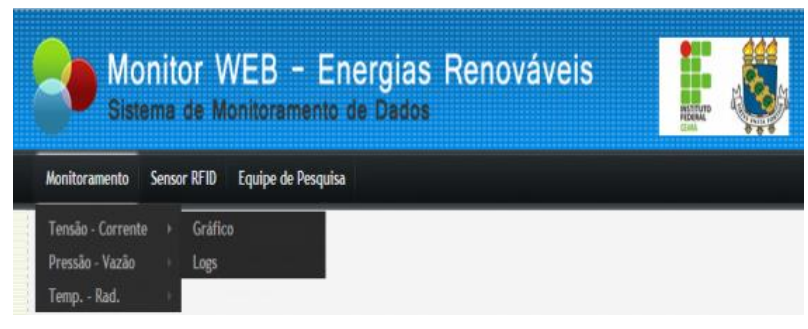

Fig. 8. Menu screen expanded.

To access the system remotely via a computational device, the static IP defined in the code must be entered, as mentioned, e. g. 192.168.1.195, followed by a slash and the commands programmed in the microcontrolled circuit (192.168.1.195/YT), as shown in Figure 9, along with the confirmation screen of thermoelectric microgenerator data measuring by the online server.

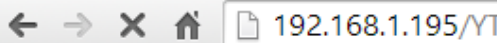

HTTP/1.0 Host: dns1 HTTP/1.1200 OK Content-Type: text/html

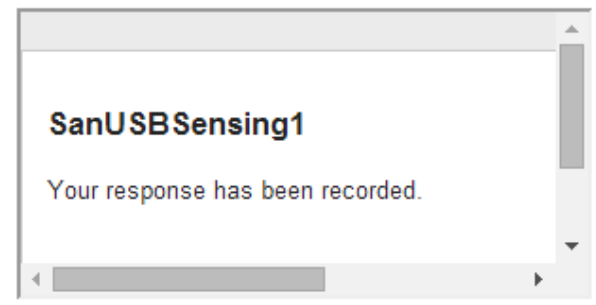

Access $\underline{\text { Logs }}$

Fig. 9. Confirmation response of received data by the online server. 
By accessing the "Logs" link, the user can monitor realtime information sent by the system every 10 seconds via WiFi. Generate graphics on the same page in which data are stored, is also possible, as shown in Figure 10.

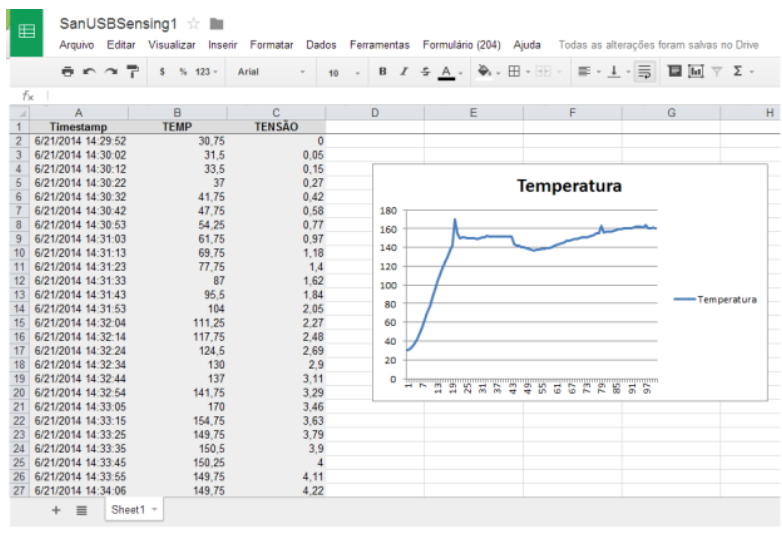

Fig. 10. Recording data in the online database and graphing.

\section{Results}

The temperature control system developed was graduated to a temperature reference of $200^{\circ} \mathrm{C}$. In one analysis, this value was reached in approximately 16 minutes as can be seen in Figure 11. The cold temperature was maintained at $40^{\circ} \mathrm{C}$, then the temperature difference was $160^{\circ} \mathrm{C}$. The temperature values were compared with the infrared temperature sensor VA6510 [26].

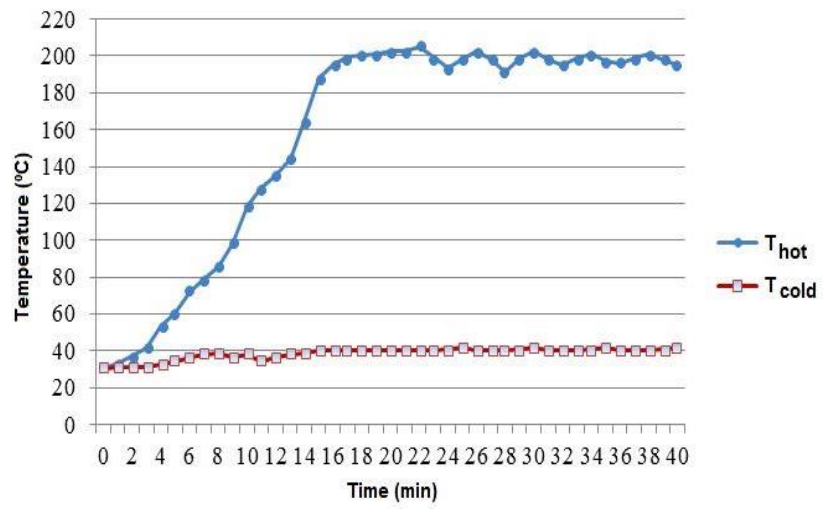

Fig. 11. Hot side and cold variation temperature.

Five different loads (10 $\Omega, 20 \Omega, 30 \Omega, 40 \Omega$ and $50 \Omega$ ) were used. For these five loads, the graph in Figure 12 shows the voltage values obtained according to the time until stabilization of the temperature on the hot side at $200^{\circ} \mathrm{C}$, i.e., after 16 minutes. The modules generate approximately $5 \mathrm{~V}$ for the different loads, however each has a different curve in time. The $50 \Omega$ load generates the maximum voltage of $5 \mathrm{~V}$ only after 15 minutes, holding at this point due to the temperature control developed, while the other reaches a peak in less time.

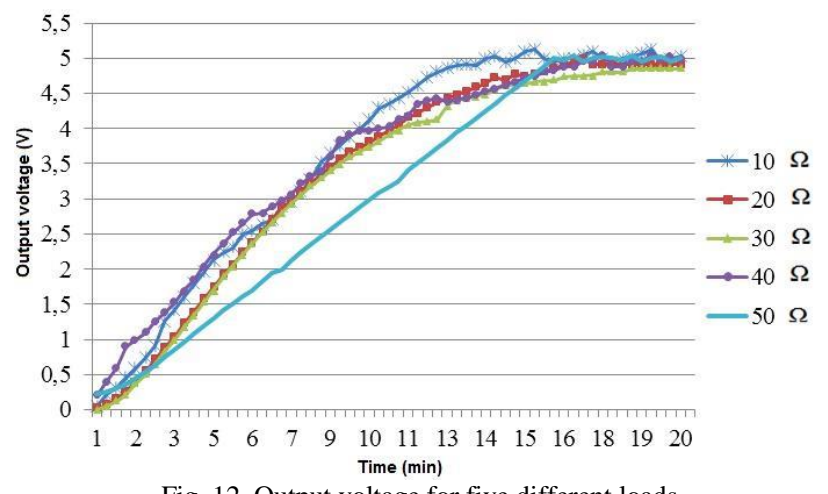

Fig. 12. Output voltage for five different loads.

\section{Conclusion}

New technologies such as thermoelectric microgeneration need for monitoring systems to collect e.g., voltage and temperature data in order to provide a more efficient study of this technology. Due to the possibility of use in recycling waste heat, this form of generation is a viable alternative, and present several advantages such as no moving parts, around 100,000 h of operational lifetime, reversible heat direction, is used in cooling and heating systems, can operate in hostile, sensitive, and smaller ambient compared to conventional cooling and are not dependent on how position are allocated in the system.

The monitoring and data acquisition system proposed was developed in open source software and multiplatform (Linux, Windows ${ }^{\circledR}$, and Mac OSX) in order to facilitate the dissemination of the computational tool developed for different user profiles. The open source monitoring software proposed enables monitoring the microgeneration plant via smartphones, tablets or other mobile devices with Internet access. As a case study for the monitoring system, four thermoelectric microgenerators (TEGs) in series were used.

The online database can be queried by any computing device connected to the Internet via password. Queries can be made at any time by updating the database every 10 minutes, which is usually the maximal time step for data acquisition systems of renewable energy plants.

The WEB monitoring and designed data acquisition system of the microgeneration plant was efficient because of the online query possibility and real-time operation of the electrical microgeneration plant, showing a behavior according to the project. The use of tools based on open source software for online monitoring applied to microgeneration systems allows greater accessibility to general users.

The proposed online monitoring and data acquisition model can be expanded to record data from other systems with analog or digital sensors, as well as other types of data from microgeneration plants using renewable energy sources. 


\section{Acknowledgement}

The authors would like to thank CNPq and Vale for the financial support through the Forma-Engenharia program, CAPES and Deutscher Akademischer Austauschdienst (DAAD) for research grants awarded, and UFC and IFCE for the availability of laboratories and equipment.

\section{References}

[1] D. M. Rowe, G. Min, "Design Theory of Thermoelectric Modules for Electrical Power Generation", in IEEE Proc. Science Measurement Technology 1996, Issue 6, pp. 351356.

[2] E. Schwyter, W. Glatz, L. Durrer, C, Hierold, "Flexible micro thermoelectric generator based on electroplated Bi2+xTe3-x", in Proc. DTIP of MEMS \& MOEMS 2008, pp. 9-11.

[3] H. S. Han, Y. H. Kim, S. Y. Kim, S. Um, J. M. Hyun, "Performance Measurement and Analysis of a Thermoelectric Power Generator", in Proc. IEEE Thermal and Thermomechanical Phenomena in Electronic Systems 2010, pp. 1-7.

[4] E. Schwyter, W. Glatz, L. Durrer, C. Hierold, "Bi2Te3Based flexible micro thermoelectric generator with optimized design", J. Microelectromech 2009, Vol. 18, pp. 763-772.

[5] A. Kucukkomurler, "Thermoelectric Power high temperature wireless sensing", J. Thermal Sci. and Techn. 2009, Vol. 4, pp. 63-73.

[6] B. Jang, S. Seungwoo Han, J. Kim, "Optimal design for micro-thermoelectric generators using finite element analysis", Microelectronic Engineering 2011, Vol. 88, 775778.

[7] R. Y. Nuwayhid, D. M. Rowe, G. Min, "Low cost stove-top thermoelectric generator for regions with unreliable electricity supply", Renew. Energy 2003, Vol. 28, pp. 205 222.

[8] P. H. Kao, P. J. Shih, C. L. Dai, M. C. Liu, "Fabrication and Characterization of CMOS-MEMS Thermoelectric Micro Generators", Sensors 2010, Vol. 10, pp. 1315-1325.

[9] S. Lineykin, S. Ben-Yaakov, "Modeling and Analysis of Thermoelectric Modules", IEEE Transactions on Industry Applications 2007, Vol. 43, Issue 2, pp. 505-512.

[10] NASA'S JET PROPULSION LABORATORY, MultiMission Radioisotope Thermoelectric Generator Heat Exchangers for the Mars Science Laboratory Rover, NASA Tech Briefs (2012).

[11] S. C. S. Jucá, P. C. M. Carvalho, R. I. S. Pereira, D. Petrov and U. Hilleringmann, "Design and Implementation of a High Temperature Control Monitoring Applied to Micro Thermoelectric Generators", International Conference on Renewable Energies and Power Quality (ICREPQ 2013), Vol.11.
[12] R. Ahiska, S. Dislitas, "Microcontroller Based Thermoelectric Generator Application", G. U. J. of Sci. 2006, Vol. 19, pp. 131-146.

[13] J. A. Paradiso, T. Starner, "Energy scavenging for mobile and wireless electronics", IEEE Energy Harvesting \& conservation 2005, Vol. 4.1, pp. 18-27.

[14] D. M. Rowe, "Thermoelectrics, an environmentally-friendly source of electrical power", Renewable Energy 1999, Vol. 16, pp. 1251-1256.

[15] F. Stabler, "Automotive applications of high efficiency thermoelectrics", In Proc. of DARPA/ONR Program Review and DOE High Efficiency Thermoelectric Workshop 2002, pp. 1-26.

[16] J. Torresen, Y. Hafting, K. Nymoen, "A New Wi-Fi based Platform for Wireless Sensor Data Collection", Available online:

http://folk.uio.no/jimtoer/NIME2013_JimTorresen.pdf (accessed on 25 Jan 2014).

[17] D. Krizaj, M. Baloh, R. Brajkovic, T. Zagar, "Design and Development of a Portable WiFi enabled BIA device", Journal of Physics 2013, Conference Series 434.

[18] G. C. Martins, F. R. de Sousa, "An RF-Powered Temperature Sensor Designed for Biomedical Applications", Integrated Circuits and Systems Design, 26th Symposium on Integrated Circuits and Systems Design, SBCCI 2013.

[19] M. S. Durante, S. Mahlknecht, "An Ultra Low Power Wakeup Receiver for Wireless Sensor Nodes", The Third International Conference on Sensor Technologies and Applications, SENSORCOMM 2009, pp. 167 -170.

[20] H. Raisigel, G. Chabanis, I. Ressejac, M. Trouillon, "Autonomous Wireless Sensor Node for Building Climate Conditioning Application", Fourth International Conference on Sensor Technologies and Applications, SENSORCOMM 2010, pp. 68-73.

[21] G. U. Gamm, S. Sester, L. M. Reindl, "SmartGateconnecting wireless sensor nodes to the Internet", J. Sens. Sens. Syst. 2013, Vol. 2, pp. 45-50.

[22] SanUSB Group, SanUSB tool, Available online: $\mathrm{http} / / / \mathrm{www}$. tinyurl.com/SanUSB (accessed on 02 Oct 2014).

[23] J. W. Paulson, G. Succi, A. Eberlein, "An empirical study of open-source and closed-source software products", IEEE Transactions on Software Engineering 2004, Vol. 30, pp. 246-256.

[24] G. S. V. Radha, K. Rao, and G. Radhamani, WiMAX: A Wireless Technology Revolution, Auerbach Publications, New York (2007)

[25] IEEE802, Official IEEE 802.11 working group timelines, Available online: http://www.ieee802.org/11/Reports/ 802.11_Timelines.htm (accessed on 04 Apr 2014).

[26] V\&A INSTRUMENT, Mini-infrared Thermometer, User Manual (2010). 